\title{
Who you calling obese, Doc?
}

Previously published at www.cmaj.ca

$\mathrm{T}$ he language of medicine, like any profession's lexicon, changes over time. Sometimes the evolution is for the better. As medicine has become less paternalistic, and the public more educated about personal health, communication between doctors and patients has become more open and direct. Other times, however, medical terminology is changed for reasons other than clarity to avoid offence, to lessen stigma, to reduce fear.

Though lapsing into euphemism can soften a bleak diagnosis, it can also lead to confusion. Euphemism is no friend of precision. Thus doctors are sometimes faced with a dilemma: Should I be sensitive or accurate?

Consider the word "obesity." It is a clinical term, applicable to a person whose body mass index (BMI) is 30 $\mathrm{kg} / \mathrm{m}^{2}$ or higher. In most Western nations, about $20 \%$ of people fall into this category. Obesity even has a code in the International Statistical Classification of Diseases.

But the word, like many medical terms, has entered the vernacular — with the stigma firmly attached. Because of the association, people tend to use the words "obese" and "obesity" with care. Some want to get rid of them altogether.
For example, the city council of Liverpool, England, is considering substituting the phrase "unhealthy weight" for "obesity" in all literature aimed at children.

Doctors are also aware that patients don't like to be labelled as obese, even if the label is accurate. Dr. Arya Sharma, chair for cardiovascular obesity research and management at the University of Alberta in Edmonton, says he never refers to a patient as an obese individual, which implies they are defined by their condition. Instead, he will use phrases such as "person with obesity." He also prefaces the word "obese" with "medically."

"When you put the word 'medically' in front of it, it implies you are talking about a medical condition," says Sharma, who is also the scientific director of the Canadian Obesity Network.

In a recent paper, Sharma explored historical approaches to classifying obesity. Some descriptive terms once used in medicine, he noted, were far from kind (Int J Obes 2009;33:28995). "Although some earlier descriptions of obesity used less pejorative synonyms such as stout and corpulent (meaning excessively fat), other terms such as mammoth, monstrous and grotesque clearly reflect societal stigmatization against the obese individual and, although the stigma remains, these terms have long been abandoned," he wrote.

Though he takes care not to offend patients, Sharma advocates for the use of the word "obesity" because it has a precise clinical definition. In fact, he insisted the word be included in the Canadian Obesity Network's name, although some feared it would repel sponsors and harm funding, and suggested names along the lines of "The Healthy Network."

Sharma also says the word "obesity" should be entered into medical records if a person's BMI is $30 \mathrm{~kg} / \mathrm{m}^{2}$ or higher. To just record the BMI would be akin to recording blood pressure without noting the presence of hypertension.

Some health experts believe the stigma associated with certain conditions can have a positive effect, serving as a powerful motivator for people to improve their health. But when it comes to obesity, Sharma says, stigma does nothing but harm. It can deter people from seeking medical care and lead to depression, anxiety, poor body image and suicidal thoughts.

"These are concrete health risks," says Sharma. "It's not just about not being kind to people."

Efforts to abolish the stigma surrounding some health problems, however, are little more than feel-good rhetoric — and a waste of time -
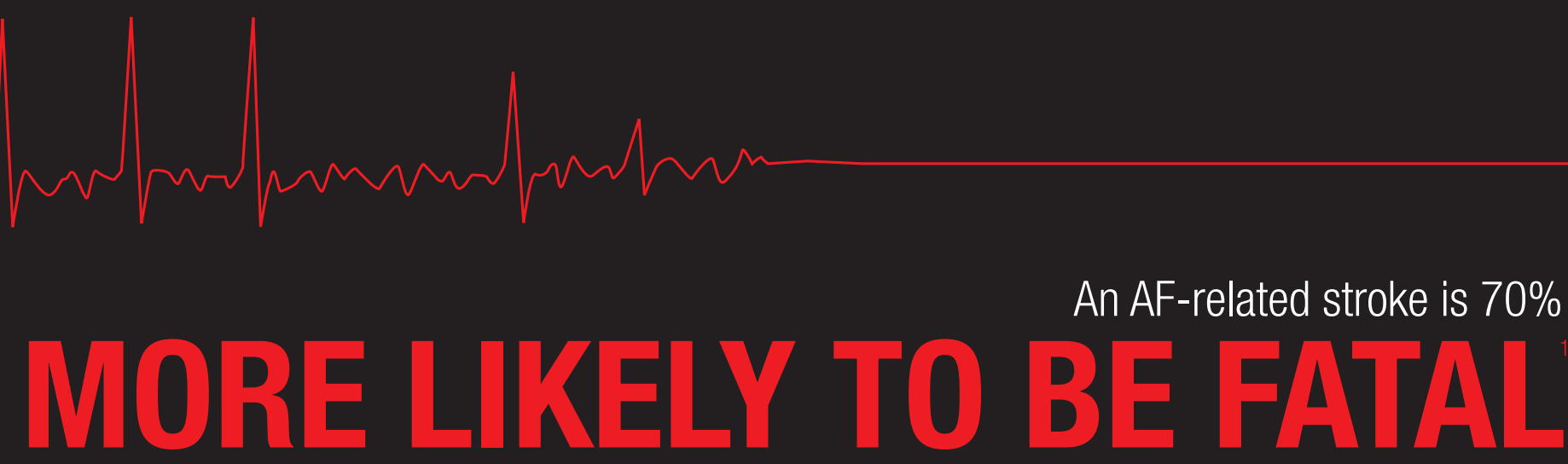


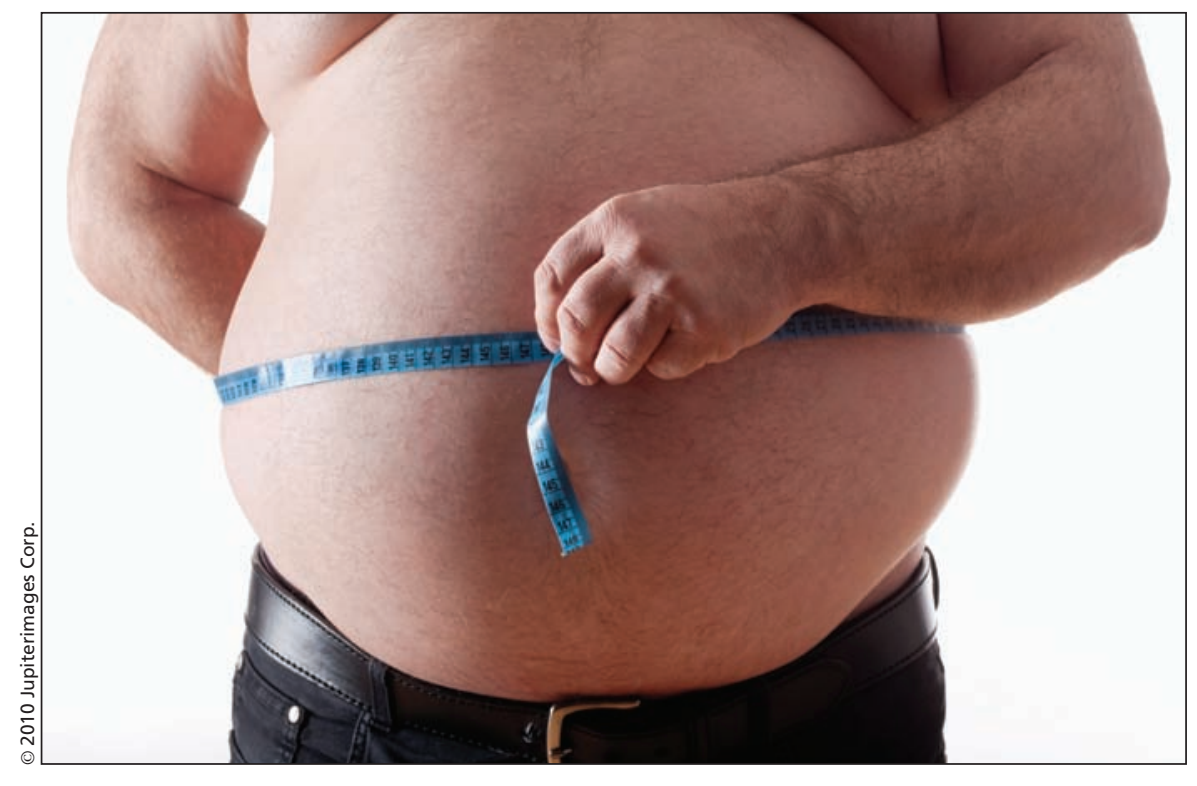

Some doctors say it's more sensitive to preface the word "obese" with the word "medically." Obesity is a clinical term, applicable to a person whose body mass index is 30 $\mathrm{kg} / \mathrm{m}^{2}$ or higher.

according to Dr. Sally Satel, a psychiatrist and resident scholar at the American Enterprise Institute in Washington, DC. Once, during a talk on addiction, Satel says, someone was offended that she used the word "addict." The belief that using a different word will destigmatize something that society frowns upon is naive, says Satel. "You can change the word and the new word becomes loaded. Then you are shifting your vocabulary to outrun the offence."

Linguists refer to this as the "euphemism treadmill." A new word is chosen to refer to a sensitive topic and soon that word becomes tainted, prompting people to search for yet another word. But linguistic gymnastics, most language experts agree, do little to dispel stigma.

Furthermore, Satel says, addiction is a behaviour deserving of stigma. In an article titled "In Praise of Stigma," she argued that stigma reflects a societal norm and can motivate people to change their behaviour (www.aei.org/article /27282). Though "stigma abolitionists" have good intentions, for the most part, Satel wrote that the fear of attributing blame for destructive behaviour can itself be destructive. There is much about addiction that is voluntary, she writes, and to dispel the concept of willpower will only deter the recovery process.

"There is nothing unethical — and everything natural and socially adaptive - about condemning the reckless and harmful behaviours that addicts commit," she wrote. "This need not negate our sympathy for them or our duty to provide care."

The fear of offending someone can drive language into foggy territory. This is just as true in medicine as in the rest of society, says Satel, who in 2001 published a book called $P C, M . D$. : How Political Correctness Is Corrupting Medicine. Twisting language to spare someone's feelings can sometimes be more insulting than edifying, says Satel. "You are basically sending the message that people are so fragile that they can't tolerate reality."

Medical language can also be distorted through the efforts of a group or movement with an agenda. Satel notes that in mental health, the word "consumer" is often used instead of "patient." This came about because of the campaigning of the antipsychiatry movement, says Satel. "First off, doctors treat patients. In some ways, this is an affront to our profession. It shows a veiled contempt for expertise."

The shift from "patient" to "client" to "consumer" is also the fault of health care providers, says Dr. Allison Crawford, an assistant professor of psychiatry at the University of Toronto in Ontario.
Health care has become corporatized, she says, and the language of business is permeating the profession of medicine.

"In clinical practice, especially if it's multidisciplinary, if you use the word 'patient' in meetings, people literally cringe," says Crawford.

In 2008, she wrote a paper exploring the use of euphemisms to describe taboo topics such as death ("gone to sleep"), pregnancy loss ("born still”) and menstruation ("time of the month"). Historically, euphemisms have also been used in medicine to link diseases to external sources. "Early terms for syphilis often linked the disease with foreigners - Spanish pox, Neapolitan bone ache, malady of France" (www .chass.utoronto.ca/ cpercy/courses/6362 -CrawfordAllison.htm).

A desire to deflect blame for errors has also lead to vague terms such as "adverse event" and "poor outcome."

Another reason doctors sometime prefer indirect language is to ease patient worries. Researchers found that doctors in the United Kingdom tend to avoid the term "heart failure" because it implies a sense of hopelessness and patient culpability (Patient Educ Counc 2005;57:321-6). Instead, doctors favoured euphemisms such as "fluid on your lungs as your heart is not pumping hard enough."

Patients informed of their conditions with euphemisms reported feeling more hopeful about the consequences their illness would have on their lives.

In a $B M J$ editorial, it was suggested that "cardiac impairment" would be a "kinder, and more accurate" alternative to "heart failure" (BMJ 2005;331:415-6). The article drew many positive letters, in which people suggested other terms, such as "heart weakness" and "Cardiovascular Insufficiency Syndrome."

One letter, however, made the case for more drastic change. "It is still not quite in tune with the "politically correct' culture of the last couple of decades. We would suggest a more appropriate, and current, term, 'cardiac deferred success.' It really implies no blame to anyone: much better." Roger Collier, CMAJ

DOI:10.1503/cmaj.109-3308 